\title{
Self-aggregated nanoparticles based on amphiphilic poly(lactic acid)-grafted-chitosan copolymer for ocular delivery of amphotericin B
}

This article was published in the following Dove Press journal:

International Journal of Nanomedicine

26 September 2013

Number of times this article has been viewed

\author{
Wenjun Zhou' \\ Yuanyuan Wang ${ }^{2}$ \\ Jiuying Jian ${ }^{2}$ \\ Shengfang Song' \\ 'Department of Ophthalmology, \\ Yongchuan Hospital, Chongqing \\ Medical University, Chongqing, \\ People's Republic of China; ${ }^{2}$ College \\ of Life Science, Chongqing Medical \\ University, Chongqing, People's \\ Republic of China
}

Background: The purpose of this study was to develop a self-aggregated nanoparticulate vehicle using an amphiphilic poly(lactic acid)-grafted-chitosan (PLA-g-CS) copolymer and to evaluate its potential for ocular delivery of amphotericin B.

Methods: A PLA-g-CS copolymer was synthesized via a "protection-graft-deprotection" procedure and its structure was confirmed by Fourier transform infrared spectroscopy, ${ }^{1} \mathrm{H}$ nuclear magnetic resonance, and X-ray diffraction spectra. Amphotericin B-loaded nanoparticles based on PLA-g-CS (AmB/PLA-g-CS) were prepared by the dialysis method and characterized for particle size, zeta potential, and encapsulation efficiency. Studies of these AmB/PLA-g-CS nanoparticles, including their mucoadhesive strength, drug release properties, antifungal activity, ocular irritation, ocular pharmacokinetics, and corneal penetration were performed in vitro and in vivo.

Results: Fourier transform infrared spectroscopy, ${ }^{1} \mathrm{H}$ nuclear magnetic resonance, and X-ray diffraction spectra showed that the PLA chains were successfully grafted onto chitosan molecules and that crystallization of chitosan was suppressed. The self-aggregated PLA-g-CS nanoparticles had a core-shell structure with an average particle size of approximately $200 \mathrm{~nm}$ and zeta potentials higher than $30 \mathrm{mV}$. Amphotericin B was incorporated into the hydrophobic core of the nanoparticles with high encapsulation efficiency. Sustained drug release from the nanoparticles was observed in vitro. The ocular irritation study showed no sign of irritation after instillation of the PLA-g-CS nanoparticles into rabbit eyes. The minimal inhibitory concentration of the AmB/PLA-g-CS nanoparticles showed antifungal activity similar to that of free amphotericin B against Candida albicans. The in vivo ocular pharmacokinetic study suggested that the PLA-g-CS nanoparticles have the advantage of prolonging residence time at the ocular surface. The corneal penetration study showed that the PLA-g-CS nanoparticles could penetrate into the cornea.

Conclusion: Our results suggest that this nanoparticulate vehicle based on a PLA-g-CS copolymer might be a promising system for effective ocular delivery of amphotericin B.

Keywords: chitosan, poly(lactic acid), nanoparticles, amphotericin B

\section{Introduction}

Fungal keratitis, a corneal fungal infection of the eye caused mainly by Candida species, has become the leading cause of blindness resulting from corneal disease in the People's Republic of China. ${ }^{1}$ Amphotericin B is a macrocyclic polyene antibiotic with a broad antifungal spectrum and remains the drug of first choice for fungal infections caused by yeast. ${ }^{2,3}$ Currently, there are no licensed topical formulations containing amphotericin $\mathrm{B}$, and extemporaneous preparations of amphotericin B as eye drops $(0.1 \%-0.3 \%)$ from available marketed parenteral formulations (Fungizone ${ }^{\circledR}$, Bristol-Myers Squibb,
Correspondence: Shengfang Song

Department of Ophthalmology,

Yongchuan Hospital, Chongqing

Medical University, 439 Xuanhua Road,

Yongchuan District, Chongqing 402460,

People's Republic of China

Tel $+86238538 \quad 1640$

Fax +86238538I623

Email cqsongshengfang@163.com 
New York, NY, USA) are used frequently in the clinic. However, this type of amphotericin B formulation usually has poor clinical efficacy in the treatment of fungal keratitis for several reasons. First, due to the critical and pharmacokinetically specific environment that exists in the eye, topical drug delivery is complicated by effective removal mechanisms, including the blink reflex, tear turnover, and limited drug contact time. ${ }^{4,5}$ Second, the human cornea, which comprises epithelium, the substantia propria, and endothelium, restricts the ocular entry of drug molecules. ${ }^{6,7}$ Amphotericin B has a high molecular mass (924.10 Da), so cannot easily penetrate the intact corneal epithelium. ${ }^{8}$ Moreover, frequent application of amphotericin B eye drops containing deoxycholate is irritating to the cornea, which would decrease patient compliance, causing treatment failure and emergence of drug resistance..$^{3,9}$ As a result of these factors, the bioavailability of the drug is extremely low. Only 1\%-5\% of the applied active ingredient is available to the ocular tissue and the rest would reach the systemic circulation via the conjunctiva and tear flow in the nasal passage, with undesirable side effects. ${ }^{10}$

To overcome these therapeutic gaps, many studies have focused on the scope for developing suitable antifungal preparations using novel delivery systems such as collagen shield, liposome, and polymer nanoparticles. ${ }^{2,11-13}$ Although there have been some advances, the best treatment remains uncertain. In view of its biocompatible, biodegradable, and nontoxic properties, chitosan, a hydrophilic biopolymer, is a proposed material with a good application potential for ocular drug delivery. ${ }^{14}$ Additionally, formulations of chitosan or chitosan-coated nanoparticles are reported to have the ability to prolong the corneal residence time of encapsulated drugs considerably and to enhance transcorneal delivery of drugs. ${ }^{15-18}$ However, because of its hydrophilic properties, chitosan is not a suitable carrier for hydrophobic drugs such as amphotericin $\mathrm{B}(\log P=0.8) \cdot{ }^{13}$ Hydrophobically modified chitosan is a type of amphiphilic polymer formed when a chitosan molecule chain is grafted by special hydrophobic groups. Amphiphilic polymers have attracted increasing interest in the pharmaceutical arena because of their unique structure. Compared with chitosan, amphiphilic chitosan contains both hydrophobic and hydrophilic segments in the same chain, and can self-aggregate due to its intramolecular and/or intermolecular hydrophobic interactions when dissolved in water. Using this process, polymer nanoparticles with a hydrophobic core and a hydrophilic shell can be prepared, whereby the hydrophobic microenvironment formed by association of the hydrophobic components enables the nanoparticles to act as a reservoir for hydrophobic drugs. ${ }^{19,20}$
The purpose of the present work was to demonstrate the feasibility of amphiphilic chitosan self-aggregated nanoparticles as a hydrophobic drug carrier for ocular delivery of amphotericin B.

Hydrophobically modified chitosan was synthesized using poly(lactic acid) (PLA) as a graft material. PLA, a linear aliphatic thermoplastic polyester, is a synthetic biodegradable copolymer with good mechanical properties. PLA nanoparticles have been investigated as a carrier for drug delivery and have shown good properties in terms of sustained drug release and protection of drugs against degradation over a relatively long period of time. ${ }^{21,22}$ Selfaggregating amphotericin B-loaded nanoparticles based on a PLA-graft-chitosan copolymer (AmB/PLA-g-CS) were prepared and characterized. In vitro studies of these AmB/ PLA-g-CS nanoparticles were performed to determine their mucoadhesive strength, drug release, and antifungal activity. Ocular irritation was evaluated by the Draize method. The ocular pharmacokinetics of the topically administered nanoparticles was investigated in New Zealand White rabbits. Fluorescein isothiocyanate (FITC)-labeled nanoparticles were used to investigate the potential for corneal penetration.

\section{Materials and methods Materials}

Chitosan (95\% deacetylated), PLA, 2, 4-tolylene diisocyanate, and phthalic anhydride were purchased from Huayi Biotech Company (Shanghai, People's Republic of China). New Zealand White rabbits were obtained from the Animal Center at Chongqing Medical University (Chongqing, People's Republic of China). Candida albicans was a gift from the Microbiology Laboratory at Chongqing Medical University. All other materials, including amphotericin B, were obtained from Sigma-Aldrich (St Louis, MO, USA).

\section{Synthesis of PLA-g-CS copolymer}

The PLA-g-CS copolymer was synthesized via a "protection-graft-deprotection" procedure as previously described. ${ }^{23}$ First, chitosan was added to a solution of phthalic anhydride in dried dimethylformamide, and the mixture was heated in nitrogen at $120^{\circ} \mathrm{C}$ with stirring for 8 hours. Phthaloyl chitosan (PHCS) was obtained as a yellow powdery material and the degree of substitution of phthaloyl groups was determined to be $98 \%$ from the $\mathrm{C} / \mathrm{N}$ value of elemental analysis. Secondly, 4-tolylene diisocyanate-terminated prepolymers were prepared by dropping 4-tolylene diisocyanate into PLA solution at an $\mathrm{NCO} / \mathrm{OH}$ 
ratio of $2: 1(\mathrm{~mol} / \mathrm{mol})$. The reactions were performed under nitrogen at $60^{\circ} \mathrm{C}$ for 30 minutes, using dimethylformamide as a solvent. The above prepolymers were then mixed with PHCS in dimethylformamide at different mass ratios of PLA/ PHCS $(2: 1,4: 1,6: 1)$. The reaction was continued with stirring at $90^{\circ} \mathrm{C}$ for 3 hours under nitrogen. The obtained product was poured into iced water and separated by filtration. The unreacted PLA-NCO was removed by Soxhlet extraction with acetone for 24 hours. Finally, the obtained phthaloylprotected graft copolymer was stirred in dimethylformamide and heated to $100^{\circ} \mathrm{C}$. The reaction was continued for 2 hours to deprotect the phthaloyl groups. The solution was cooled down to room temperature and then poured into ethanol for precipitation. The CS-g-PLA precipitate was collected and washed thoroughly with ethanol and dried. The graft content was calculated as follows:

$$
\% \mathrm{G}=100 \times\left(\mathrm{W}_{\mathrm{g}}-\mathrm{W}_{0}\right) / \mathrm{W}_{0}
$$

where $\mathrm{W}_{\mathrm{g}}$ and $\mathrm{W}_{0}$ are the weight of the graft copolymers and PHCS, respectively.

\section{Characterization of PLA-g-CS copolymer}

Fourier transform infrared transmission spectra were obtained from samples using a FTIR-8900 device (Shimadzu, Kyoto, Japan) by the $\mathrm{KBr}$ method. ${ }^{1} \mathrm{H}$ nuclear magnetic resonance (NMR) spectra were recorded on a $300 \mathrm{NMR}$ spectrometer (Avance, Karlsruhe, Germany) in $\mathrm{d}_{6}$-dimethylsulfoxide. Powder X-ray diffraction diagrams were recorded using a D/ max2500 X-ray diffractometer (Rigaku, Tokyo, Japan) with graphite-monochromatized $\mathrm{Cu}-\mathrm{K}$ radiation $(\mathrm{k}=1.54 \mathrm{~A})$.

\section{Preparation of AmB/PLA-g-CS nanoparticles}

The amphotericin B-loaded nanoparticles were prepared by the dialysis method. ${ }^{24}$ Briefly, amphotericin B and PLAg-CS copolymer were dissolved in dimethylsulfoxide. The solution was stirred at room temperature for 1.5 hours and then dialyzed against deionized water for 24 hours using a dialysis bag (molecular weight cutoff 3,500). Distilled water was exchanged every hour for the first 4-hour period and every 4 hours for the next 20 hours. The resulting solution was centrifuged at $10,000 \mathrm{rpm}$ and $4^{\circ} \mathrm{C}$ for 1 hour. The precipitate was collected and washed thoroughly with acetone and dried. Empty nanoparticles were prepared by the same procedure described above with the exception of amphotericin B. The encapsulation efficiency (EE) and loading efficiency (LE) were determined by ultraviolet analysis after dissolving dried amphotericin B-loaded nanoparticles in dimethylsulfoxide according to the following respective equations:

$\% \mathrm{EE}=100 \times($ amount of amphotericin $\mathrm{B}$ in nanoparticles $/$ total amount of added amphotericin B)

$\% \mathrm{LE}=100 \times($ amount of amphotericin B in nanoparticles $/$ total amount of formulation components).

The morphology of the nanoparticles was examined using a transmission electron microscope (Tecnai-10, Philips, Tokyo, Japan). Samples of the nanoparticle suspension $(5-10 \mu \mathrm{L})$ were dropped onto a carbon film-coated 200 mesh copper grid and allowed to dry in air for 10 minutes. The samples were stained with $1 \%$ (w/v) aqueous uranyl acetate solution for 1.5 minutes, and any excess uranyl acetate was removed with filter paper before viewing under the transmission electron microscope.

The mean size, size distribution (polydispersity index) and zeta potential of the nanoparticles were determined by photon correlation spectroscopy and laser Doppler anemometry, respectively, using a Zetasizer Nano ZS90 (Malvern Instruments, Malvern, UK).

\section{In vitro studies of AmB/PLA-g-CS nanoparticles}

\section{Mucoadhesive strength}

In order to estimate the mucoadhesive force of the AmB/ PLA-g-CS nanoparticles, a mucoadhesion test was performed by measuring the change in zeta potential on interaction with negatively charged mucin, as described elsewhere. ${ }^{25}$ Equal volumes of $0.1 \%(\mathrm{w} / \mathrm{v})$ mucin solution and nanoparticles were vortexed for one minute and the zeta potential of the mixture was measured. Alteration of the zeta potential of the nanoparticles indicated an interaction with mucin.

\section{In vitro drug release}

Amphotericin B has very poor aqueous solubility. In order to increase its solubility and maintain sink conditions, $1 \%$ (v/v) Tween 80 in phosphate-buffered saline, $\mathrm{pH}$ 7.4, was used as a dissolution medium. An in vitro drug release experiment was carried out as follows. Ten milligrams of lyophilized drug-loaded nanoparticles were reconstituted in $10 \mathrm{~mL}$ of phosphate-buffered saline and then introduced into dialysis tubes (molecular weight cutoff 12,000). The dialysis tubes were placed in a $200 \mathrm{~mL}$ bottle with $95 \mathrm{~mL}$ of phosphate-buffered saline, and the medium was stirred at $37^{\circ} \mathrm{C}$. At different time intervals, the medium was taken 
and diluted with dimethylsulfoxide, and the concentration of amphotericin B released was determined using an ultravioletspectrophotometer (Shimadzu). The freshly prepared $0.15 \%$ (w/v) amphotericin B was also determined under similar conditions and considered as the reference.

\section{In vitro antifungal activity}

Free amphotericin B, empty nanoparticles, and amphotericin B-loaded nanoparticles were examined for antifungal activity by testing against $C$. albicans, according to Clinical and Laboratory Standards Institute document M27-A3 (2008) guidelines. ${ }^{26}$ Samples of free amphotericin B, empty nanoparticles, and amphotericin B-loaded nanoparticles were dissolved in dimethylsulfoxide and added to Roswell Park Memorial Institute (RPMI) growth medium with L-glutamine and morpholinopropanesulfonic acid (MOPS) buffer ( $\mathrm{pH} 7.0$ ). A total volume of $100 \mu \mathrm{L}$ of RPMI growth medium was placed in each well of a 96-well plate containing serial dilutions of amphotericin B concentrations (0.03-16 $\mu \mathrm{g} / \mathrm{mL})$. Cell suspensions of $C$. albicans were prepared in RPMI 1640 medium and adjusted to give a final inoculums concentration of $2 \times 10^{3}$ colony-forming units per $\mathrm{mL}$. The fungal suspension $(100 \mu \mathrm{L})$ was added to each well and the trays were incubated at $35^{\circ} \mathrm{C}$ for 48 hours in ambient air. The minimum inhibitory concentration was read as the lowest antifungal concentration with completely no turbidity (100\% growth reduction) compared with growth in the antifungal-free growth well for all agents.

\section{In vivo studies}

\section{of AmB/PLA-g-CS nanoparticles}

\section{Ocular irritation}

A modified Draize test was performed in order to evaluate the toxicity of the amphotericin B-loaded nanoparticles. Samples of the nanoparticles $(10 \mu \mathrm{L})$ were instilled into the right eye of the New Zealand White rabbit by gently pulling the lower lid away from the eyeball to form a cup. The left eye served as the control and was treated with phosphate-buffered saline ( $\mathrm{pH}$ 7.4). The lids were then gently held together for 10 seconds and released. The blink rate, discomfort (redness and irritation), discharge rate, and swelling of the cornea and conjunctiva were examined for one day at regular intervals of $1,3,6,8,12$, and 24 hours.

\section{Ocular pharmacokinetics}

The ocular pharmacokinetics of amphotericin B administered in the different formulations (free amphotericin B and amphotericin B-loaded nanoparticles) was evaluated at a dose strength of $0.15 \%(\mathrm{w} / \mathrm{v})$. Briefly, $20 \mu \mathrm{L}$ of each formulation was instilled into the lower conjunctival sac of the right cornea in each rabbit using a micropipette without actually touching the eye and irritating the corneal surface. Tear samples $(10 \mu \mathrm{L})$ were withdrawn without anesthesia using microcapillaries at appropriate intervals over a 4-hour period. The microcapillaries were then emptied and diluted. After centrifugation for 10 minutes at $10,000 \times g$, the supernatant was evaporated under a stream of nitrogen. The residues were redissolved with $100 \mu \mathrm{L}$ of the mobile phase for analysis by high-pressure liquid chromatography. The assay conditions were as follows: mobile phase, $10 \mathrm{mM}$ acetate buffer, $\mathrm{pH} 7.2$, and acetonitrile (63:37); flow rate $1.2 \mathrm{~mL}$ per minute; and detection wavelength $408 \mathrm{~nm}$.

\section{Corneal penetration study}

The PLA-g-CS nanoparticles were labeled by adding a specified amount of FITC in dimethylsulfoxide and then processed via the same methods as for preparation of amphotericin B-loaded nanoparticles (final FITC concentration $0.03 \%, \mathrm{w} / \mathrm{w})$. FITC was also directly dissolved in phosphate-buffered saline to a concentration of $0.03 \%$ (w/w) and taken as reference. New Zealand White rabbits were placed in restraint boxes and four $15 \mu \mathrm{L}$ instillations of the FITC-loaded nanoparticles were topically administered in the inferior conjunctival cul-de-sac of the right eye at 5-minute intervals, while FITC solution was instilled in the left eye as the control. At $0.5,1$, and 3 hours after the last instillation, the eyes were washed with phosphate-buffered saline and the rabbits were sacrificed. The corneas were then excised, embedded in optimal cutting temperature medium, and frozen at $-22^{\circ} \mathrm{C}$, then sectioned into $8 \mu \mathrm{m}$ and observed under an inverted fluorescence microscope (IX71, Olympus, Tokyo, Japan). The in vivo studies complied with the university animal ethics committee guidelines. This research was approved by the university animal ethics committee (No CQMU120213.05).

\section{Statistical analysis}

Statistical analyses were undertaken using Origin 8.0 software. Data were compared using the Student's $t$-test, and a one-way analysis of variance with a Bonferroni post-test was used for group analysis. Ocular pharmacokinetic parameters of amphotericin B in lacrimal fluid were derived from the tear concentration-time profile using WinNonlin 5.1. The results are expressed as the mean and standard deviation. Statistical significance was considered at a probability of $P<0.05$. 


\section{Results}

\section{Synthesis and characterization of PLA-g-CS copolymer}

The procedure used to synthesize the PLA-g-CS copolymer is shown in Figure 1. The amino groups on the chitosan backbone were first protected by phthalic anhydride. PHCS was obtained and its structure was confirmed by Fourier transform infrared spectroscopy. The spectrum in Figure 2B clarifies that PHCS shows critical signals at $1,777,1,712$, and $721 \mathrm{~cm}^{-1}$, assigned to the carbonyl group, the tertiary amino group, and the aromatic ring of the phthalimido group, respectively. The PLA chains were then grafted onto PHCS via the reaction between $\mathrm{OH}$ groups on PHCS and the terminated NCO groups on prepolymer. Finally, the N-phthaloyl groups were deprotected by incubation with hydrazine to bring active amino groups back to chitosan. The spectrum in Figure $2 \mathrm{C}$ shows that, compared with PHCS, the peaks at 1,712, 1,777, and $721 \mathrm{~cm}^{-1}$ almost disappeared, while new peaks at 1,538 and $1,758 \mathrm{~cm}^{-1}$ come out in the spectrum of PLAg-CS, indicating formation of an amide ester linkage (-OCONH-) and ester groups of PLA branches. This confirms that the PLA chains were grafted onto chitosan successfully.

Figure $3 \mathrm{~A}$ shows the ${ }^{1} \mathrm{H}$ NMR spectrum of the PLA-g-CS copolymer. The strong signals at $2.5 \mathrm{ppm}$ and $3.4 \mathrm{ppm}$ belong to the solvent $\mathrm{d}_{6}$-dimethylsulfoxide and $\mathrm{H}_{2} \mathrm{O}$, respectively. Peak (a) at $1.4 \mathrm{ppm}$ is caused by the methyl protons of the PLA side chain. Peak (b) at 5.2 ppm should be assigned to the $-\mathrm{CH}$-hydrogen of the lactyl moiety located on the PLA chain. These results further confirm that PLA chains had grafted onto chitosan.

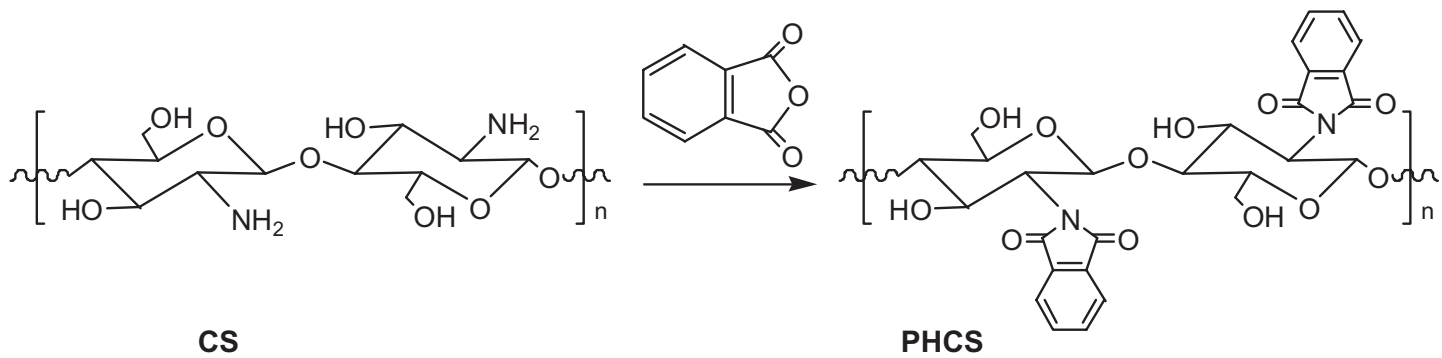<smiles>Cc1ccc(NC(=O)C(C)(C)OC(C)C(=O)C(C)(O)O)cc1[N+](=O)[O-]</smiles>

PLA

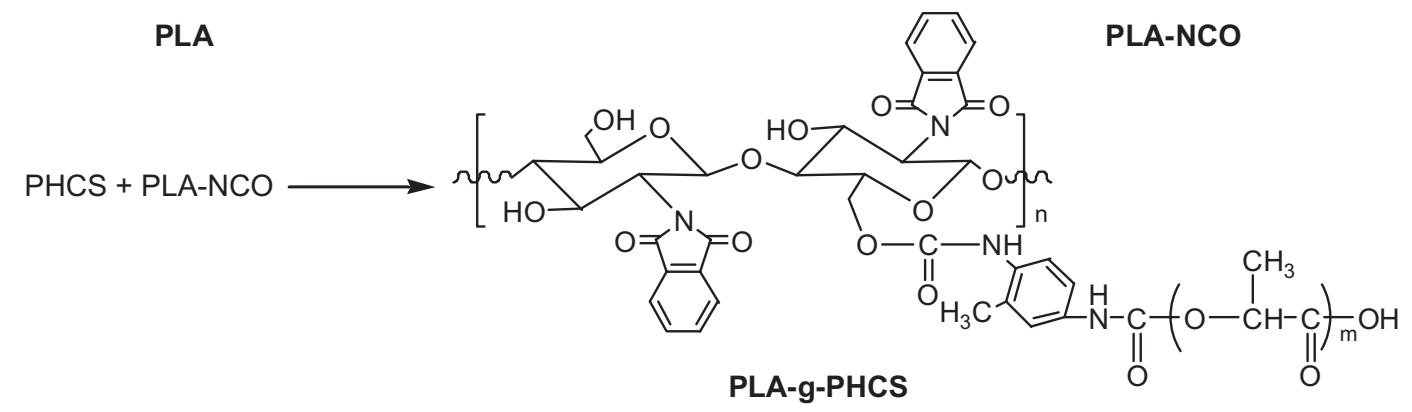

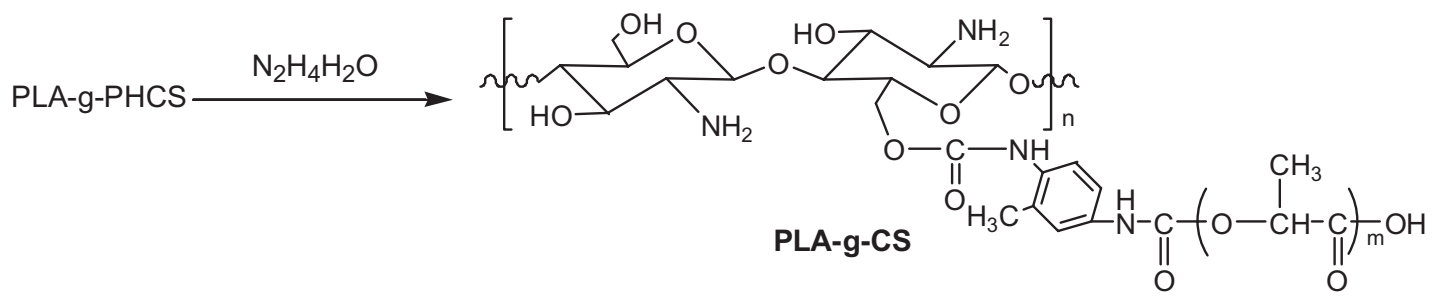

Figure I Synthetic procedure of PLA-g-CS copolymer.

Abbreviations: PLA, poly(lactic acid); CS, chitosan; PHCS, phthaloyl chitosan; PLA-g-CS, poly(lactic acid)-grafted-chitosan; PLA-NCO, TDI(NCO)-terminated poly(lactic acid); PLA-g-PHCS, poly(lactic acid)-grafted-phthaloyl chitosan. 


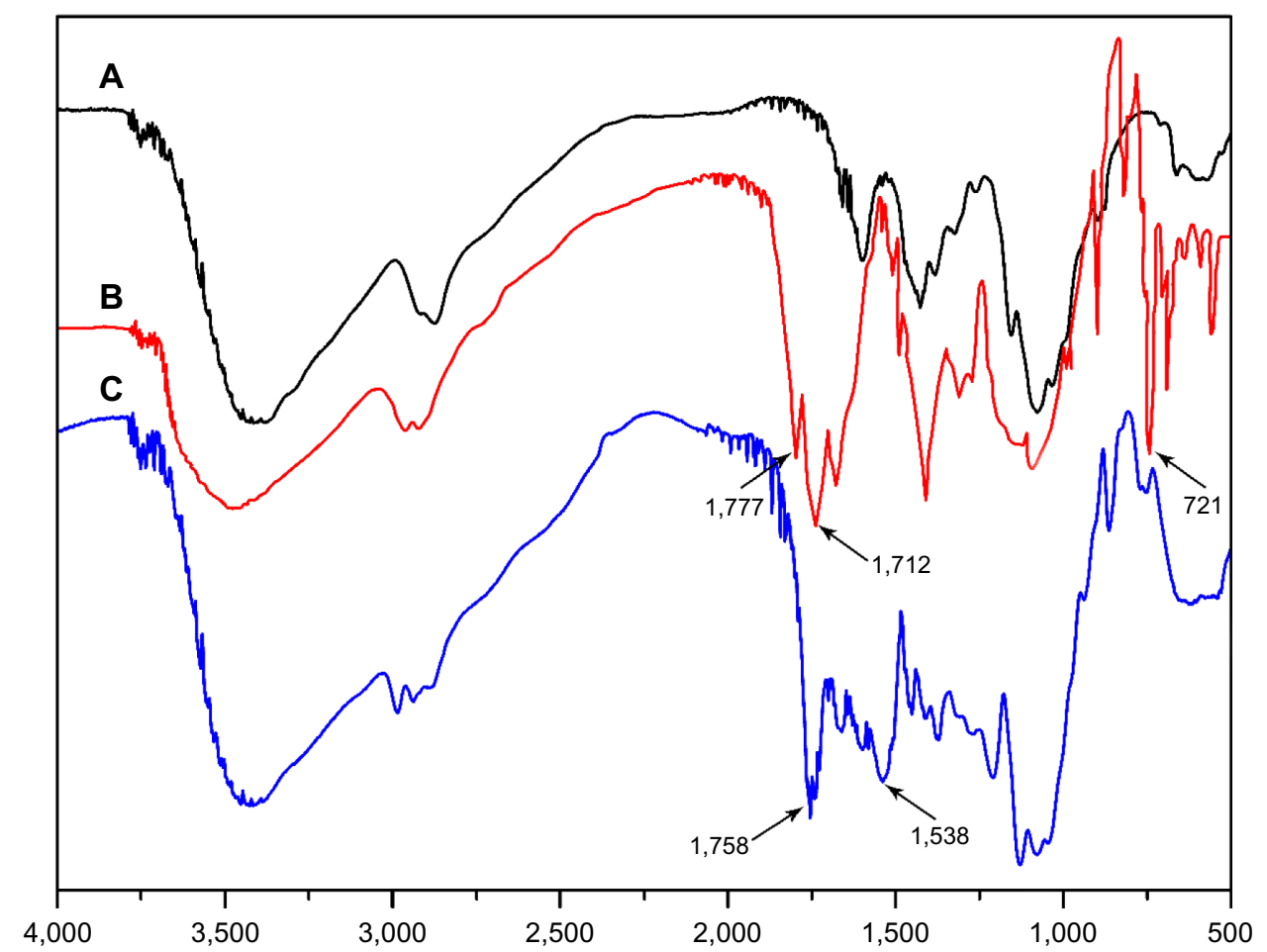

Figure 2 Fourier transform infrared spectra for CS (A), PHCS (B), and PLA-g-CS (C).

Abbreviations: PHCS, phthaloyl chitosan; CS, chitosan; PLA-g-CS, poly(lactic acid)-grafted-chitosan.
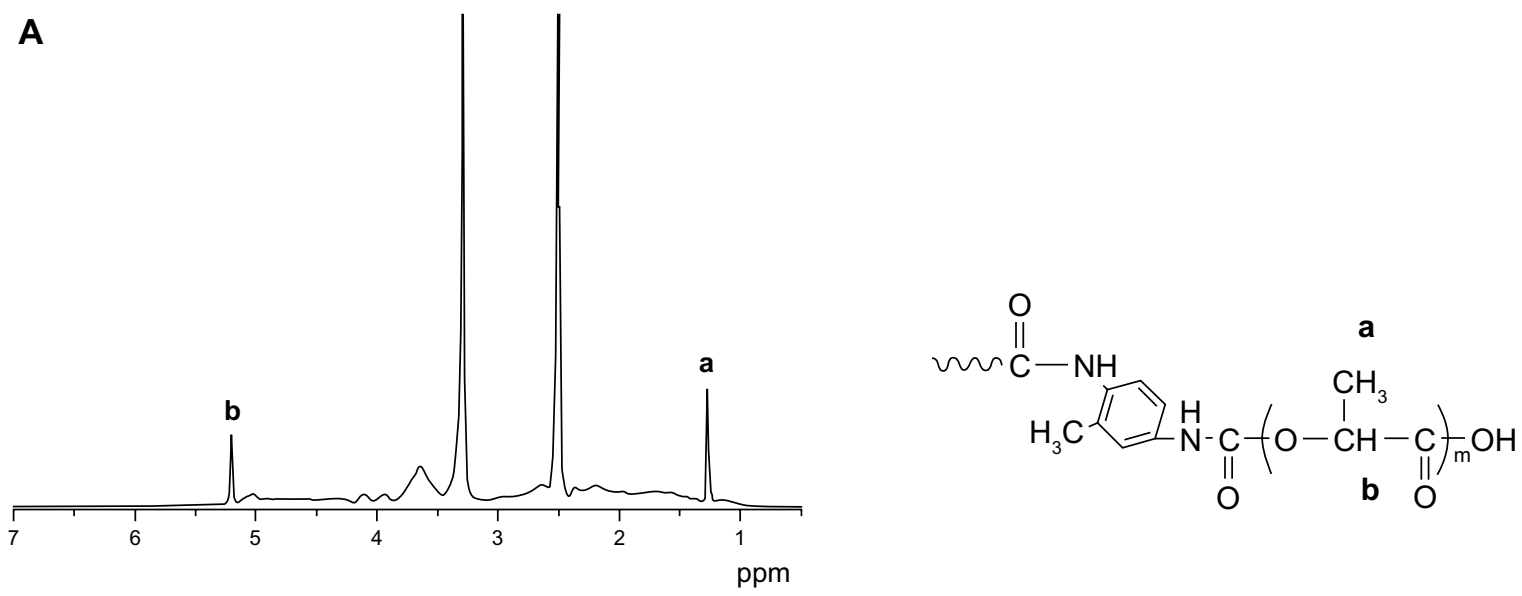

B
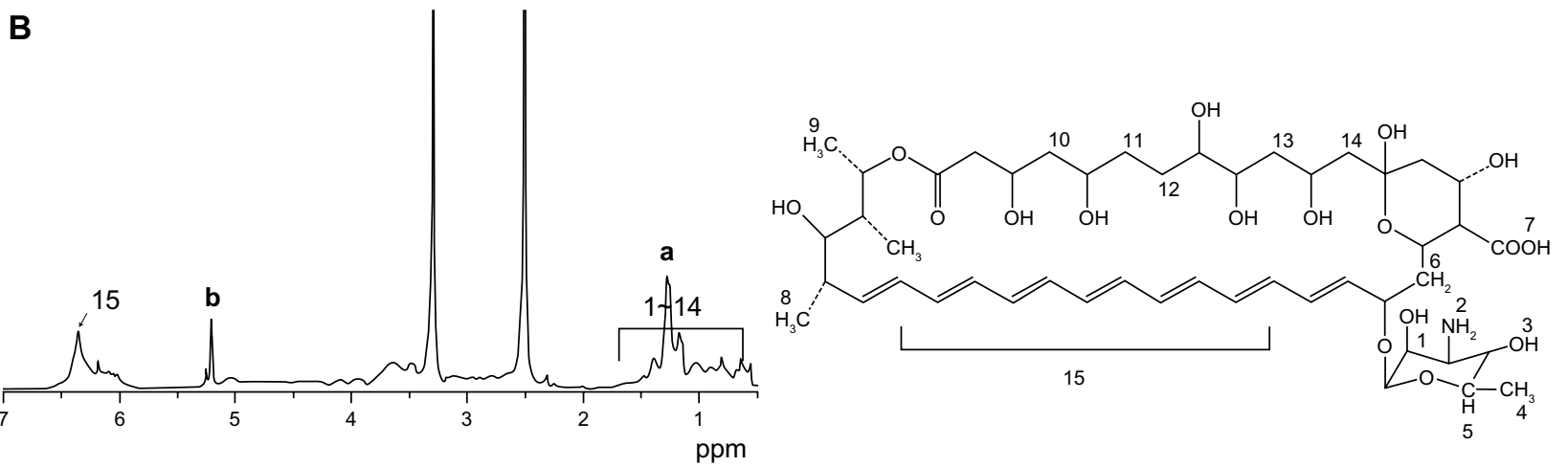

Figure 3 'H NMR spectra of PLA-g-CS copolymer (A) and AmB/PLA-g-CS nanoparticles (B).

Notes: Peak (a) represents the methyl protons of the PLA side chain. Peak (b) represents the - $\mathrm{CH}$ - hydrogen of lactyl moiety located at the PLA chains.

Abbreviations: AmB, amphotericin B; NMR, nuclear magnetic resonance; PLA-g-CS, poly(lactic acid)-grafted-chitosan. 
Figure 4 shows the X-ray powder diffraction patterns for chitosan and the PLA-g-CS copolymer. Compared with chitosan, the X-ray diffraction spectrum for PLA-g-CS shows a weaker and broader peak in the $10^{\circ}-30^{\circ}$ region. This implies that grafting of PLA with chitosan suppressed the crystallization of chitosan.

\section{Characterization of AmB/PLA-g-CS nanoparticles}

As shown in Figure 5A, amphotericin B-loaded nanoparticles were formed by self-aggregation of PLA-g-CS copolymers. Incorporation of amphotericin B into the nanoparticles was also checked by ${ }^{1} \mathrm{H}$ NMR. As shown in Figure 3B, compared with empty PLA-g-CS copolymer, intrinsic peaks of amphotericin B appeared for the amphotericin B-loaded nanoparticles, indicating that amphotericin B was encapsulated into the hydrophobic inner core of the PLA-g-CS copolymer nanoparticle. Figure 5B shows the morphology of AmB/ PLA-g-CS nanoparticles. The nanoparticles have a spherical shape and a diameter of about $200 \mathrm{~nm}$. The characteristics of AmB/PLA-g-CS nanoparticles with different PLA/PHCS feed ratios are summarized in Table 1. As shown, a higher feeding amount of PLA caused a higher graft content in the PLA-g-CS copolymer. The grafting content could be beyond $100 \%$ when the PLA/PHCS feed ratio was only $4: 1$ (w/w). However, as the graft content became higher, the particle size of nanoparticles increased, with a range of approximately 150-260 nm. AmB/PLA-g-CS nanoparticles had a positive zeta potential greater than $30 \mathrm{mV}$ that was not affected by the change in PLA/PHCS feed ratio. The nanoparticles showed

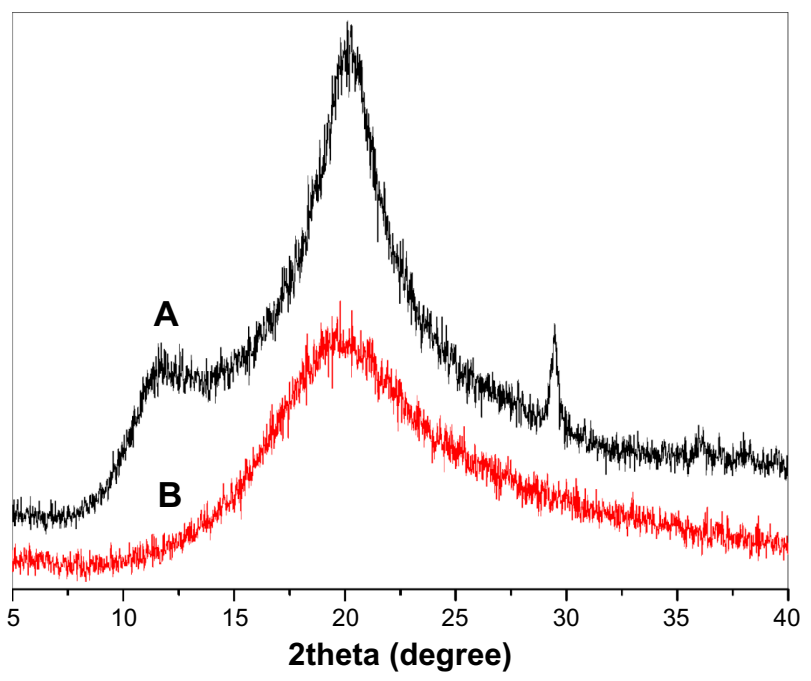

Figure 4 X-ray diffraction of CS (A) and PLA-g-CS copolymer (B). Abbreviations: PLA-g-CS, poly(lactic acid)-grafted-chitosan; CS, chitosan. a high EE of up to $70 \%$ in each group. As the PLA/PHCS feed ratio increased, the EE increased.

\section{Mucoadhesive strength}

Figure 6 shows the changes in surface charge of the AmB/ PLA-g-CS nanoparticles with different PLA/PHCS feed ratios after mixing with mucin. The surface charge of the nanoparticles decreased after incubation with mucin in all groups. This reduction could be due to ionic interaction between negatively charged sialic groups in mucin and the positively charged surface layer of the AmB/PLA-g-CS nanoparticles. The reduction in zeta potential of the nanoparticles before and after mixing indicate that the nanoparticles may have a strong mucoadhesive force towards the ocular surface.

\section{In vitro drug release}

In vitro drug release of the freshly prepared $0.15 \%(\mathrm{w} / \mathrm{v})$ amphotericin B and AmB/PLA-g-CS nanoparticles with different PLA/PHCS feed ratios in simulated ocular circumstances $\left(37^{\circ} \mathrm{C}, \mathrm{pH} 7.4\right)$ was studied. As shown in Figure 7 , the release profile of $0.15 \%(\mathrm{w} / \mathrm{v})$ amphotericin B was rapid; approximately $53.7 \%$ of the amphotericin B was released within 0.5 hours and $100 \%$ within 4 hours. In comparison with amphotericin B solution, the AmB/PLA-g-CS nanoparticles released their drug content more slowly and showed a two-step release pattern, ie, one initial burst release of about 30\%-40\% of amphotericin B, followed by a second slow-release phase sustained for up to 11 hours. In addition, the drug release rate decreased as PLA/PHCS feed ratios was increased.

\section{In vitro antifungal activity}

To evaluate the antifungal activity of the amphotericin B-loaded nanoparticles, the minimum inhibitory concentration of free amphotericin B, amphotericin B-loaded nanoparticles, and empty PLA-g-CS copolymer nanoparticles was tested with C. albicans. As shown in Table 2, the minimum inhibitory concentration of the amphotericin B-loaded nanoparticles was similar to that of free amphotericin B, while the empty polymeric nanoparticles had a higher minimum inhibitory concentration value, indicating that amphotericin B-loaded nanoparticles have an antifungal potential similar to that of free amphotericin B, but the PLA-g-CS copolymer did not affect the antifungal activity of the polymeric nanoparticles.

\section{Ocular irritation}

The results of the irritation test are summarized in Table 3. In all cases, the hourly blink rate of the rabbits was found 

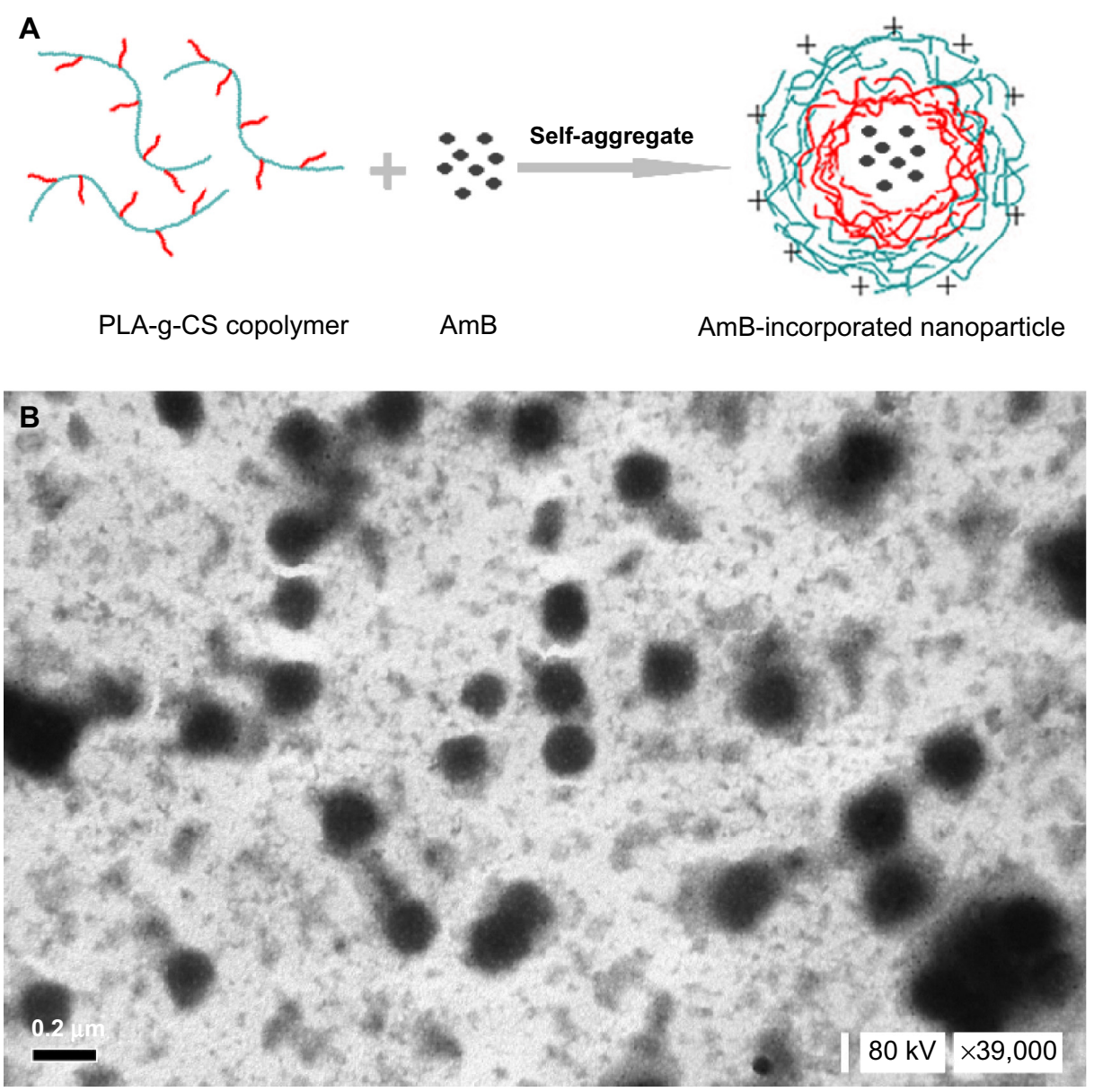

Figure 5 Schematic diagram of self-aggregation formed by the PLA-g-CS copolymer in aqueous medium (A) and transmission electron micrograph of AmB/PLA-g-CS nanoparticles (B) (PLA/PHCS feed ratios=4:I).

Abbreviations: AmB, amphotericin B; PHCS, phthaloyl chitosan; PLA, poly(lactic acid); PLA-g-CS, poly(lactic acid)-grafted-chitosan.

to be increased only in the first hour and then returned to normal (five blinks per hour). The temporary increase in blink rate may have been due to the low temperature of the samples when taken from the $4^{\circ} \mathrm{C}$ refrigerator. According to Draize grading, the scored value was zero for both phosphatebuffered saline and the nanoparticles. No signs of irritation or damaging effects to the ocular tissues in rabbit eyes was observed after instillation of the nanoparticles.

\section{Ocular pharmacokinetics}

The ocular pharmacokinetics of amphotericin B administered as free amphotericin B solution were compared with those of the AmB/PLA-g-CS nanoparticle formulation in the New Zealand White rabbit (Figure 8 and Table 4). As shown in Table 4, the area under the concentration-time curve for the AmB/PLA-g-CS nanoparticle formulation was significantly increased compared with that of free amphotericin B solution, which was 1.95 -fold higher. An increase in the half-life $\left(\mathrm{t}_{1 / 2}\right)$ of amphotericin B was seen in the nanoparticle formulation by up to 1.5 -fold. The maximum concentration of the nanoparticle formulation decreased significantly when compared with that of the free amphotericin B solution. Clearance was also decreased significantly and the mean residence time was significantly increased in the nanoparticle formulation

Table I Characteristics of AmB/PLA-g-CS nanoparticles with different PLA/PHCS feed ratios (mean \pm standard deviation, $\mathrm{n}=3$ )

\begin{tabular}{lllllll}
\hline PLA:PHCS ratio & \%G & Particle size $(\mathbf{n m})$ & Zeta potential $(\mathbf{m V})$ & PI & \%EE & \%LE \\
\hline $2: 1$ & 67.25 & $152.00 \pm 3.8$ & $35.96 \pm 0.8$ & $0.26 \pm 0.04$ & $70.65 \pm 3.18$ & $18.24 \pm 0.8$ \\
$4: 1$ & 103.64 & $196.20 \pm 1.9$ & $36.50 \pm 0.6$ & $0.25 \pm 0.03$ & $76.87 \pm 3.08$ & $21.20 \pm 1.3$ \\
$6: 1$ & 162.16 & $266.63 \pm 2.8$ & $36.85 \pm 0.4$ & $0.21 \pm 0.03$ & $81.63 \pm 3.24$ & $22.12 \pm 1.5$ \\
\hline
\end{tabular}

Abbreviations: \%G, per cent graft content; PI, polydispersity index; EE, encapsulation efficiency; LE, loading efficiency; AmB, amphotericin B; PHCS, phthaloyl chitosan; PLA, poly(lactic acid); PLA-g-CS, poly(lactic acid)-grafted-chitosan. 


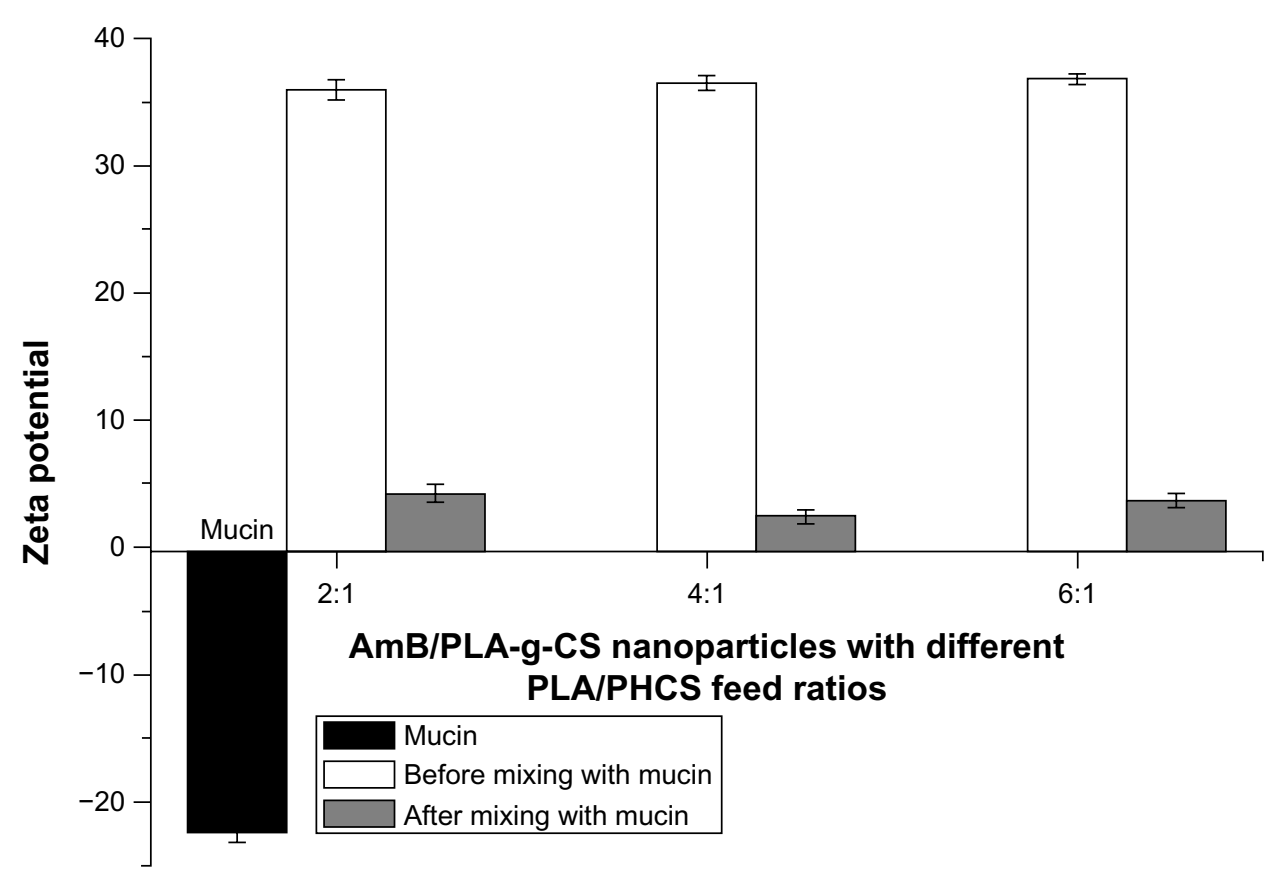

Figure 6 Zeta potential values obtained for mucin and AmB/PLA-g-CS nanoparticles before and after incubation with mucin (mean \pm standard deviation, $n=3, P<0.05$ ). Abbreviations: AmB, amphotericin B; PLA, poly(lactic acid); PLA-g-CS, poly(lactic acid)-grafted-chitosan; PHCS, phthaloyl chitosan.

as compared with the free amphotericin B solution. These results indicate that the AmB/PLA-g-CS nanoparticles could prolong drug contact time and prevent pulsed drug release on the ocular surface, further confirming the mucoadhesiveness of the nanoparticles.

\section{Corneal penetration}

The polymeric nanoparticles were labeled with encapsulated FITC to study their potential ability to transport the drug into the cornea. Fluorescence microscopic images of the treated corneas are shown in Figure 9. From fluorescence

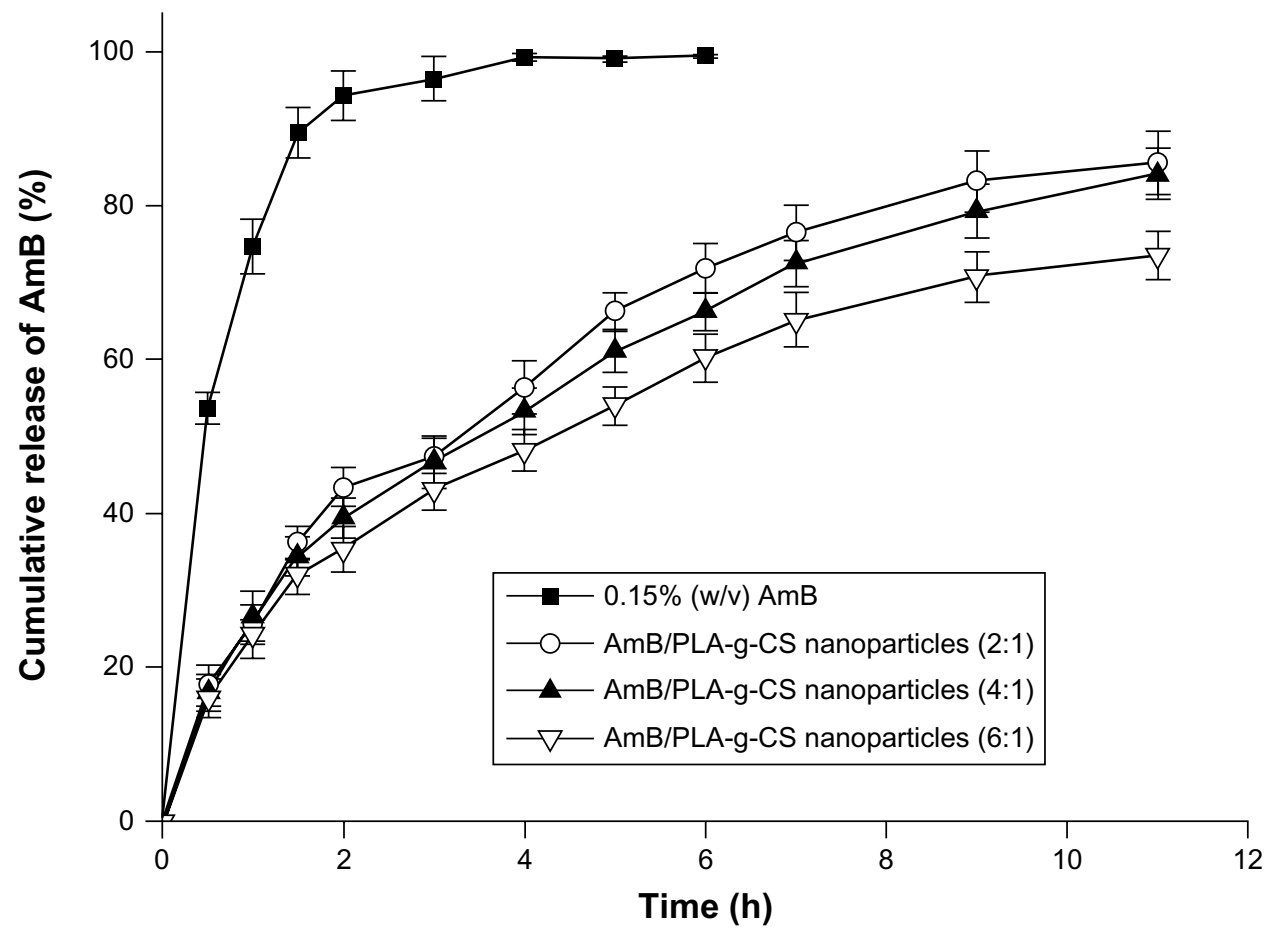

Figure 7 Cumulative release of AmB from $0.15 \%$ (w/v) AmB and AmB/PLA-g-CS nanoparticles with different PLA/PHCS feed ratios (mean \pm standard deviation, $n=5$ ). Abbreviations: AmB, amphotericin B; PLA, poly(lactic acid); PHCS, phthaloyl chitosan; PLA-g-CS, poly(lactic acid)-grafted-chitosan. 
Table 2 Effect of free AmB and AmB-loaded nanoparticles on MIC against Candida albicans

\begin{tabular}{ll}
\hline & MIC $(\mu \mathrm{g} / \mathrm{mL}, \mathbf{n}=\mathbf{3})$ \\
\hline Free AmB & 0.96 \\
AmB-loaded nanoparticles $^{\mathrm{a}}$ & 0.96 \\
Empty nanoparticles $^{\mathrm{a}}$ & $>10.0$ \\
\hline
\end{tabular}

Note: ${ }^{a} \mathrm{AmB}$-loaded or empty nanoparticles with PLA/PHCS feed ratios $(4: 1)$ were used for this test.

Abbreviations: $\mathrm{AmB}$, amphotericin $\mathrm{B}$; MIC, minimum inhibitory concentration; PHCS, phthaloyl chitosan; PLA, poly(lactic acid).

microscopy, it can be seen that both types of FITC can reach the cornea after instillation to inferior conjunctival cul-desac. However, the polymeric nanoparticles showed markedly enhanced permeation and retention effects compared with the FITC solution. For both the FITC solution and the polymeric nanoparticles, fluorescence could only be detected at the epithelial site during the initial 0.5 hours. With the passage of time, the fluorescence signal increased gradually at the inner site of the cornea when treated with polymeric nanoparticles, and the entire corneal area was stained with fluorescence by 3 hours. This result indicates that the polymeric nanoparticles have the ability to enhance penetration of the drug into the cornea.

\section{Discussion}

When treating fungal keratitis, clinical efficacy would depend on the concentration of antifungal agent achieved in corneal tissue. The cornea is relatively inaccessible to systemically administered drugs and, as a result, topical drug delivery remains the preferred route in most cases. Considering the effective removal mechanisms and the barriers operating in the eye, an ideal ocular drug delivery system for amphotericin B must be able to remain in the precorneal area for a prolonged period of time, be capable of sustaining drug release, and improve drug penetration. The preparation of PLA-grafted-chitosan and its aggregation behavior have been well demonstrated in previous reports..$^{23,27,28}$ Given that this polymeric nanoparticle has a distinctive structure and may meet the above-mentioned requirements, its application as an ocular carrier for amphotericin B was investigated in this study.
It has been reported that the amino groups on the chitosan molecule are very important for maintaining the various specific functions of chitosan, including biological activity and cationic polymer properties. ${ }^{29,30}$ Therefore, the synthesis of PLA-g-CS copolymer was carried out via a "protection-graft-deprotection" procedure. As shown in Figure 1, this method enables the successful grafting of the PLA chain on the chitosan molecule and, at the same time, ensures preservation of the amino groups on the chitosan molecule. Chitosan is insoluble in water, but can dissolve in dilute acid. However, when PLA chains are introduced, the crystallinity of chitosan could be destroyed, and it was found in our study that the PLA-g-CS copolymer dissolved well in water. When dispersed in the aqueous phase, the PLA chains tend to aggregate on the inner side by hydrophobic interaction and the hydrophilic chitosan chains form on the outer layer. The transmission electron micrograph shows that the PLA-g-CS nanoparticles have a core-shell structure with a dark core region surrounding a gray color, which represents the hydrophobic portion and hydrophilic portion, respectively. For preparation of amphotericin B-loaded PLA-g-CS nanoparticles, the effect of graft content on the characteristic parameters of the nanoparticles was studied, and the results are consistent with previous findings. ${ }^{27} \mathrm{It}$ is easy to understand that, as the graft content increases, more PLA chains would aggregate in the core region and the particle size of the nanoparticles would increase. On the other hand, the increased size of the hydrophobic region would facilitate incorporation of amphotericin B, resulting in higher encapsulation efficiency. However, because the amount of chitosan chains with amino groups does not change, the zeta potential of the nanoparticles remains unaltered. It has been reported that if the zeta potential of the particles is higher than $30 \mathrm{mV}$ or lower than $-30 \mathrm{mV}$ the dispersion is stable. ${ }^{31}$ Therefore, the physicochemical stability of the nanoparticles was assessed by evaluating their particle size, zeta potential, and polydispersity index at room temperature before and after a storage period of 2 weeks. The nanoparticles showed no significant changes in these parameters, indicating that the integrity of the nanoparticles is not compromised by relatively long-term storage (data not shown).

Table 3 Testing of ocular irritation potential of different formulations using Draize scoring method $(n=3)$

\begin{tabular}{lllll}
\hline & Blink rate/hour & Discomfort/redness & Discharge & Cornea/conjunctiva \\
\hline PBS (pH 7.4) & 6 & 0 & 0 & 0 \\
Nanoparticles & 7 & 0 & 0 & 0 \\
Observations & & No discomfort and redness & No discharge & No sign of irritation and edema \\
\hline
\end{tabular}

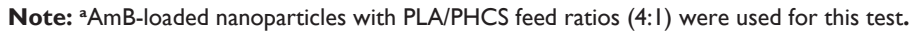

Abbreviations: AmB, amphotericin B; PBS, phosphate-buffered saline; PHCS, phthaloyl chitosan; PLA, poly(lactic acid). 


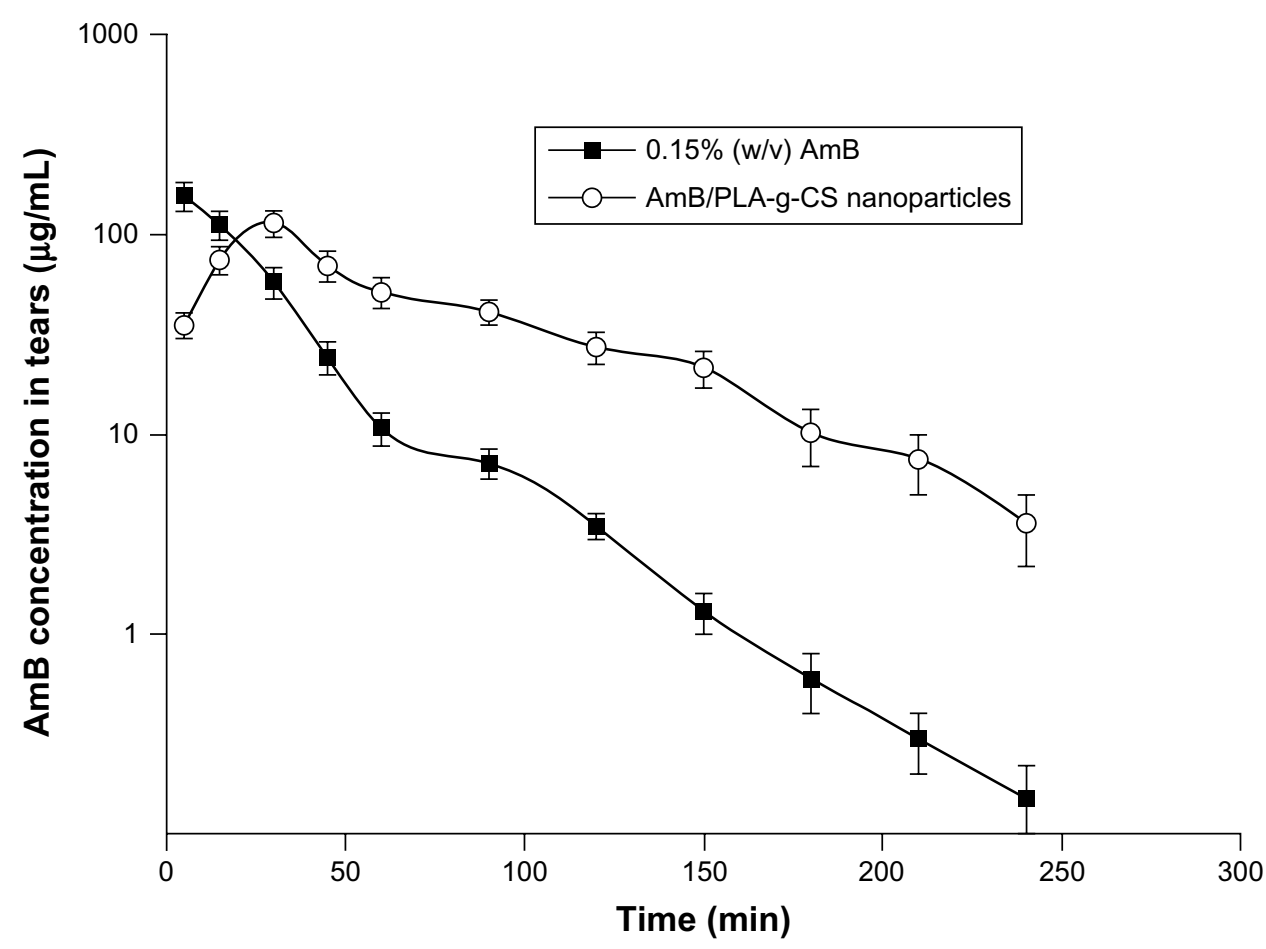

Figure 8 Tear concentration-time profile of $A m B$ administered as $0.15 \%$ (w/v) AmB and AmB/PLA-g-CS nanoparticles (mean \pm standard deviation, $n=3$ ). Abbreviations: AmB, amphotericin B; PLA-g-CS, poly(lactic acid)-grafted-chitosan.

Because of the need to prolong the drug contact time on the ocular surface, the mucoadhesive strength of the PLA-g-CS nanoparticles was investigated in vitro. Our results suggest that the PLA-g-CS nanoparticles possess mucoadhesiveness. It has been reported that the chitosan molecule can form electrostatic interactions between mucin via the $\mathrm{NH}_{3}^{+}$groups on chitosan and either $\mathrm{COO}^{-}$or $\mathrm{SO}_{3}^{-}$on the mucin carbohydrate side chain. ${ }^{32,33}$ Since the amino groups of chitosan were well preserved, the mucoadhesiveness of PLA-g-CS nanoparticles could be explained by the same mechanism. Our in vivo ocular pharmacokinetic results further demonstrate that these

Table 4 Pharmacokinetic parameters of $A m B$ in precorneal region of New Zealand White rabbits after topical instillation of AmB/PLAg-CS nanoparticles and free $A m B$ (mean \pm standard deviation, $n=3$ )

\begin{tabular}{llcc}
\hline & Units & $\begin{array}{l}\text { AmB/PLA-g-CS } \\
\text { nanoparticles }^{\mathrm{a}}\end{array}$ & Free AmB \\
\hline $\mathrm{AUC}$ & $\min \mu \mathrm{g} / \mathrm{mL}$ & $8,652.63 \pm 336.28^{\mathrm{b}}$ & $4,448.49 \pm 102.54$ \\
$\mathrm{t}_{1 / 2}$ & $\min$ & $46.57 \pm 5.49^{\mathrm{b}}$ & $30.00 \pm 4.42$ \\
$\mathrm{C}_{\max }$ & $\mu \mathrm{g} / \mathrm{mL}$ & $114.10 \pm 6.8^{\mathrm{b}}$ & $156.60 \pm 8.2$ \\
$\mathrm{Cl}$ & $\mathrm{mL} / \mathrm{min}$ & $0.0035 \pm 0.0006^{\mathrm{b}}$ & $0.0067 \pm 0.0006$ \\
MRT & $\mathrm{min}$ & $78.53 \pm 5.24^{\mathrm{b}}$ & $29.07 \pm 2.12$ \\
\hline
\end{tabular}

Notes: ${ }^{\mathrm{A} A m B}$-loaded nanoparticles with PLA/PHCS feed ratios (4:I); ${ }^{\mathrm{b} A l l}$ pharmacokinetic parameters in the nanoparticle group were significantly different compared with the free $A m B$ group $(P<0.05)$.

Abbreviations: $\mathrm{AmB}$, amphotericin B; AUC, area under the curve; $\mathrm{Cl}$, clearance; $\mathrm{t}_{1 / 2}$, half-life; $\mathrm{C}_{\max }$ maximum concentration; MRT, mean residence time; PHCS, phthaloyl chitosan; PLA, poly(lactic acid); PLA-g-CS, poly(lactic acid)-graftedchitosan. nanocarriers have an affinity for the corneal surface because the area under the concentration-time curve, $\mathrm{t}_{1 / 2}$, and mean residence time of the nanoparticles were significantly increased while clearance was significantly decreased. Covering both the corneal and conjunctival surfaces and forming part of
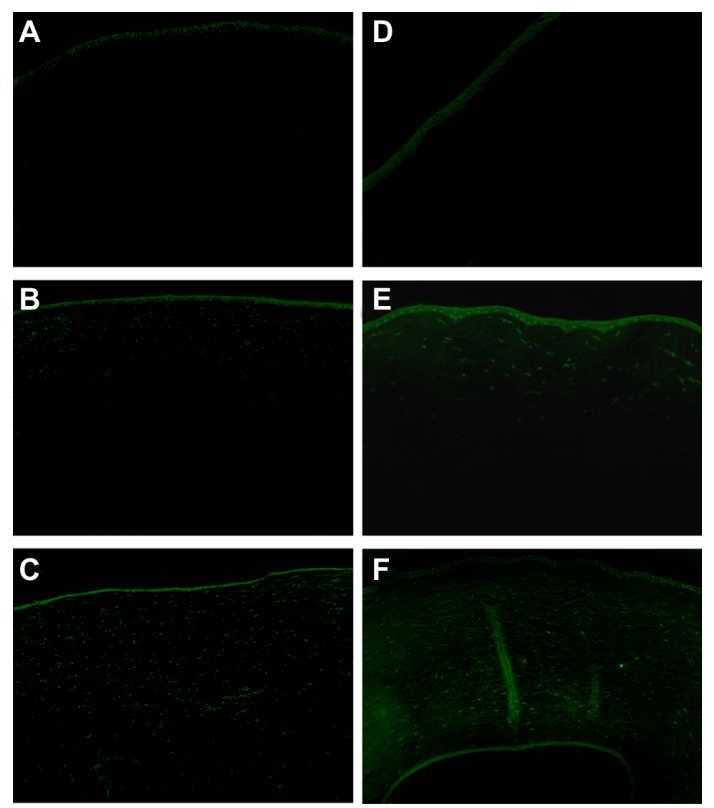

Figure 9 Fluorescence microscopy of rabbit cornea after treatment with FITC solution $(0.03 \%)$ at (A) 30 minutes, (B) I hour, and (C) 3 hours. FITC-labeled PLAg-CS nanoparticles $(0.03 \%)$ at (D) 30 minutes, (E) I hour, and (F) 3 hours.

Abbreviations: FITC, fluorescein isothiocyanate; PLA-g-CS, poly(lactic acid)-graftedchitosan. 
the tear film, is a mucin layer secreted by the goblet cells of the conjunctiva. The mucin layer is negatively charged and its turnover is thought to be relatively slow compared with the aqueous layer of the tear film. ${ }^{34}$ The administered nanoparticles would interact intimately with these extraocular structures, thus increasing the concentration and residence time of amphotericin B.

In view of the need for sustained drug release, we investigated in vitro drug release from the PLA-g-CS nanoparticles and the effect of graft content on drug release. Because amphotericin B and the inner side of the nanoparticles are both hydrophobic, incorporation of amphotericin B into the nanoparticles may be mainly due to hydrophobic interaction. Drug release from the PLA-g-CS nanoparticles showed a two-step release pattern (Figure 7). It is known that drug release from polymer particulate systems involves three different mechanisms: desorption of protein molecules from the surface of the particles; diffusion through the swollen polysaccharide matrix; and release due to polymer erosion. ${ }^{35,36}$ The first mechanism plays a major role in the burst-release phase, and the slow-release phase is due to diffusion and polymer erosion. The release rate of amphotericin B was affected by graft content of the PLA-g-CS copolymer, ie, as the graft content increased, the drug release rate decreased. This could be explained by the fact that a higher graft content of copolymer could lead to a more intensive hydrophobic interaction between the PLA chains and amphotericin B, resulting in a tighter hydrophobic core, and thus a slower rate of drug release.

The ability of the PLA-g-CS nanoparticles to penetrate the cornea was investigated with the aim of improving drug penetration. Our results indicate that the PLA-g-CS nanoparticles not only adhere to the cornea, but are also able to enter it. In fungal keratitis, fungal organisms can enter into the corneal stroma, where they slowly proliferate and destroy the surrounding tissue. ${ }^{37}$ Therefore, it is not enough to maintain an efficient antifungal therapy even if a delivery system can prolong the contact time and sustain drug release at the precorneal area. Amphotericin B is a broad spectrum antifungal agent that exerts its antifungal effects by binding to sterols in the cell membrane and damaging the membrane. ${ }^{38}$ The problem is that amphotericin B has very poor corneal penetration in the face of an intact epithelium because of its high molecular mass. O'Day et al studied the corneal penetration of topical amphotericin B, and their results showed that amphotericin B penetrated the cornea poorly in the presence of an intact corneal epithelium. However, when the corneal epithelium was removed, corneal penetration was enhanced. ${ }^{39}$ The same results were observed in a recent study, suggesting that the epithelium is a barrier to corneal penetration. ${ }^{8}$ In fact, the corneal epithelium overlying a stromal fungal infection can heal once treatment is initiated despite the presence of active infection. ${ }^{40}$ In view of this observation, an effective drug vehicle must be able to facilitate the transport of amphotericin B across the corneal epithelium. Paracellular transport (ie, through the intercellular space) and diffusion are the two mechanisms for drug transport across the cornea. ${ }^{41}$ Previous studies have demonstrated that chitosan or chitosan-coated nanocarriers can open the tight junction of corneal epithelial cells and have the ability to enhance corneal transport of certain drugs, such as cyclosporin A. ${ }^{42}$ De Campos et al systematically investigated the interaction between chitosan nanoparticles and the ocular mucosa in vivo and found that chitosan nanoparticles were preferentially transported by paracellular mechanism. The authors attributed this behavior to interaction between the positively charged amino groups of chitosan with negatively charged sites on the corneal cell surfaces and tight junctions. ${ }^{32}$ The interior of tight junctions is highly hydrated and contains fixed negative charges. In this study, the positively charged amino groups of PLA-g-CS nanoparticles may also have interacted with the tight junctions, and an alteration in the relative concentration of specific ion species in the pore volume would result in changes in tight junction resistance, which might lead to loosening or opening of the pore.

In addition, for a polymeric drug delivery to be proposed as an ophthalmic drug carrier, it is important to assay not only its biopharmaceutical properties but also its ocular tolerability. Therefore, the potential ocular irritant and/or damaging effects of the PLA-g-CS nanoparticles were evaluated. Although the biocompatibility of chitosan or PLA has been demonstrated, the ocular irritation study ensured that the developed nanoparticulate system was safe on ocular instillation. The in vitro antifungal activity of the amphotericin B-loaded nanoparticles against $C$. albicans was also investigated, and it was found that they had antifungal potential similar to that of amphotericin B. Our results demonstrate further that these PLA-g-CS copolymer-based nanoparticles can be excellent drug carriers for ocular delivery of amphotericin B.

\section{Conclusion}

In this study, we successfully demonstrated the feasibility of using PLA-g-CS nanoparticles as hydrophobic drug carriers 
for ocular delivery of amphotericin B. The unique structure of PLA-g-CS nanoparticles makes it possible to overcome the therapeutic gaps existing in ocular drug delivery. PLAg-CS nanoparticles can prolong the drug contact time, sustain drug release, and improve drug penetration. In addition, they caused no ocular irritation and demonstrated effective antifungal potential. Thus, a nanoparticulate vehicle based on a PLA-g-CS copolymer might be a useful system for effective ocular delivery of amphotericin B.

\section{Acknowledgment}

The authors thank Zuowei Yuan and Juan Liao for their assistance in conducting some of the experiments.

\section{Disclosure}

The authors report no conflicts of interest in this work.

\section{References}

1. Xie L, Zhong W, Shi W, Sun S. Spectrum of fungal keratitis in north China. Ophthalmology. 2006;113(11):1943-1948.

2. Das S, Suresh PK. Nanosuspension: a new vehicle for the improvement of the delivery of drugs to the ocular surface. Application to amphotericin B. Nanomedicine. 2011;7(2):242-247.

3. Morand K, Bartoletti AC, Bochot A, Barratt G, Brandely ML, Chast F. Liposomal amphotericin $\mathrm{B}$ eyedrops to treat fungal keratitis: physicochemical and formulation stability. Int J Pharm. 2007;344(1-2): $150-153$.

4. Kaur IP, Kakkar S. Topical delivery of antifungal agents. Expert Opin Drug Deliv. 2010;7(11):1303-1327.

5. Sahoo SK, Dilnawaz F, Krishnakumar S. Nanotechnology in ocular drug delivery. Drug Discov Today. 2008;13(3-4):144-151.

6. Eljarrat-Binstock E, Pe'er J, Domb AJ. New techniques for drug delivery to the posterior eye segment. Pharm Res. 2010;27(4):530-543.

7. Gaudana R, Jwala J, Boddu SH, Mitra AK. Recent perspectives in ocular drug delivery. Pharm Res. 2009;26(5):1197-1216.

8. Qu L, Li L, Xie H. Corneal and aqueous humor concentrations of amphotericin B using three different routes of administration in a rabbit model. Ophthalmic Res. 2010;43(3):153-158.

9. Liu Z, Li J, Nie S, Guo H, Pan W. Effects of transcutol P on the corneal permeability of drugs and evaluation of its ocular irritation of rabbit eyes. J Pharm Pharmacol. 2006;58(1):45-50.

10. Nagarwal RC, Kant S, Singh PN, Maiti P, Pandit JK. Polymeric nanoparticulate system: a potential approach for ocular drug delivery. $J$ Control Release. 2009;136(1):2-13.

11. Pleyer U, Legmann A, Mondino BJ, Lee DA. Use of collagen shields containing amphotericin B in the treatment of experimental Candida albicans-induced keratomycosis in rabbits. Am J Ophthalmol. 1992;113(3):303-308.

12. Das S, Suresh PK, Desmukh R. Design of Eudragit RL 100 nanoparticles by nanoprecipitation method for ocular drug delivery. Nanomedicine. 2010;6(2):318-323.

13. Kaur IP, Rana C, Singh H. Development of effective ocular preparations of antifungal agents. J Ocul Pharmacol Ther. 2008;24(5): 481-493.

14. Alonso MJ, Sanchez A. The potential of chitosan in ocular drug delivery. J Pharm Pharmacol. 2003;55(11):1451-1463.

15. de la Fuente M, Ravina M, Paolicelli P, Sanchez A, Seijo B, Alonso MJ. Chitosan-based nanostructures: a delivery platform for ocular therapeutics. Adv Drug Deliv Rev. 2010;62(1):100-117.
16. Enriquez de Salamanca A, Diebold Y, Calonge M, et al. Chitosan nanoparticles as a potential drug delivery system for the ocular surface: toxicity, uptake mechanism and in vivo tolerance. Invest Ophthalmol Vis Sci. 2006;47(4):1416-1425.

17. Paolicelli P, de la Fuente M, Sanchez A, Seijo B, Alonso MJ. Chitosan nanoparticles for drug delivery to the eye. Expert Opin Drug Deliv. 2009;6(3):239-253.

18. Sezer AD, Cevher E. Topical drug delivery using chitosan nano- and microparticles. Expert Opin Drug Deliv. 2012;9(9):1129-1146.

19. Elsaid N, Jackson TL, Gunic M, Somavarapu S. Positively charged amphiphilic chitosan derivative for the transscleral delivery of rapamycin. Invest Ophthalmol Vis Sci. 2012;53(13):8105-8111.

20. Zhou H, Liu X, Guo X, et al. Synthesis and characterization of amphiphilic chitosan derivatives as a nano-carrier for paclitaxel delivery. J Control Release. 2011;152 Suppl 1:e124-e125.

21. Lassalle V, Ferreira ML. PLA nano- and microparticles for drug delivery: an overview of the methods of preparation. Macromol Biosci. 2007;7(6):767-783

22. Xiao RZ, Zeng ZW, Zhou GL, Wang JJ, Li FZ, Wang AM. Recent advances in PEG-PLA block copolymer nanoparticles. Int $J$ Nanomedicine. 2010;5:1057-1065.

23. Li G, Zhuang Y, Mu Q, Wang M, Fang Ye. Preparation, characterization and aggregation behavior of amphiphilic chitosan derivative having poly (L-lactic acid) side chains. Carbohydr Polym. 2008;72(1):60-66.

24. Zhang X, Zhu X, Ke F, et al. Preparation and self-assembly of amphiphilic triblock copolymers with polyrotaxane as a middle block and their application as carrier for the controlled release of Amphotericin B. Polymer. 2009;50(18):4343-4351.

25. Zhu X, Su M, Tang S, et al. Synthesis of thiolated chitosan and preparation nanoparticles with sodium alginate for ocular drug delivery. Mol Vis. 2012;18:1973-1982.

26. Wikler M. Clinical and Laboratory Standards Institute. Performance Standards for Antimicrobial Susceptibility Testing: Eighteenth Informational Supplement. Wayne: Clinical and Laboratory Standards Institute. 2008.

27. Li J, Kong M, Cheng XJ, et al. Preparation of biocompatible chitosan grafted poly(lactic acid) nanoparticles. Int J Biol Macromol. 2012;51(3): 221-227.

28. Bhattarai N, Ramay HR, Chou SH, Zhang M. Chitosan and lactic acidgrafted chitosan nanoparticles as carriers for prolonged drug delivery. Int J Nanomedicine. 2006;1(2):181-187.

29. Amidi M, Mastrobattista E, Jiskoot W, Hennink WE. Chitosan-based delivery systems for protein therapeutics and antigens. Adv Drug Deliv Rev. 2010;62(1):59-82.

30. Agnihotri SA, Mallikarjuna NN, Aminabhavi TM. Recent advances on chitosan-based micro- and nanoparticles in drug delivery. $J$ Control Release. 2004;100(1):5-28.

31. Bihari P, Vippola M, Schultes S, et al. Optimized dispersion of nanoparticles for biological in vitro and in vivo studies. Part Fibre Toxicol. 2008;5:14.

32. de Campos AM, DieboldY, Carvalho EL, SanchezA, Alonso MJ. Chitosan nanoparticles as new ocular drug delivery systems: in vitro stability, in vivo fate, and cellular toxicity. Pharm Res. 2004;21(5):803-810.

33. Wadhwa S, Paliwal R, Paliwal SR, Vyas SP. Hyaluronic acid modified chitosan nanoparticles for effective management of glaucoma: development, characterization, and evaluation. J Drug Target. 2010;18(4):292-302.

34. Stahl U, Willcox M, Stapleton F. Osmolality and tear film dynamics. Clin Exp Optom. 2012;95(1):3-11.

35. Zhou W, Zhao M, Zhao Y, Mou Y. A fibrin gel loaded with chitosan nanoparticles for local delivery of rhEGF: preparation and in vitro release studies. J Mater Sci Mater Med. 2011;22(5): $1221-1230$.

36. Zhou S, Deng X, Li X. Investigation on a novel core-coated microspheres protein delivery system. J Control Release. 2001;75(1-2):27-36. 
37. Amescua G, Miller D, Alfonso EC. What is causing the corneal ulcer? Management strategies for unresponsive corneal ulceration. Eye (Lond). 2012;26(2):228-236.

38. Brajtburg J, Powderly WG, Kobayashi GS, Medoff G. Amphotericin B: current understanding of mechanisms of action. Antimicrob Agents Chemother. 1990;34(2):183-188.

39. O'Day DM, Head WS, Robinson RD, Clanton JA. Corneal penetration of topical amphotericin B and natamycin. Curr Eye Res. 1986;5(11): $877-882$.
40. Tuli SS. Fungal keratitis. Clin Ophthalmol. 2011;5:275-279.

41. Lawrence MS, Miller JW. Ocular tissue permeabilities. Int Ophthalmol Clin. 2004;44(3):53-61.

42. De Campos AM, Sanchez A, Alonso MJ. Chitosan nanoparticles: a new vehicle for the improvement of the delivery of drugs to the ocular surface. Application to cyclosporin A. Int J Pharm. 2001;224(1-2):159-168.

\section{Publish your work in this journal}

The International Journal of Nanomedicine is an international, peerreviewed journal focusing on the application of nanotechnology in diagnostics, therapeutics, and drug delivery systems throughout the biomedical field. This journal is indexed on PubMed Central, MedLine, CAS, SciSearch $\AA$, Current Contents ${ }^{\circledR} /$ Clinical Medicine,
Journal Citation Reports/Science Edition, EMBase, Scopus and the Elsevier Bibliographic databases. The manuscript management system is completely online and includes a very quick and fair peer-review system, which is all easy to use. Visit http://www.dovepress.com/ testimonials.php to read real quotes from published authors. 